\title{
Multidisciplinary Optimization of the Fuel Consumption of a Dual Mode Scramjet-Ramjet
}

\author{
Sean M. Torrez*, Derek J. Dalle ${ }^{*}$, James F. Driscoll ${ }^{\dagger}$ \\ University of Michigan, Ann Arbor, MI 48109
}

\begin{abstract}
Dual-mode ramjets have design requirements that are distinct from those of other high speed propulsion systems because they are expected to operate over a wide range of conditions. Several previously examined combustor designs are evaluated over a range of operating conditions using a low order model. The designs under consideration are parametrized by three variables: location of fuel injection, angle of wall divergence and location of wall divergence, and multidisciplinary optimization (MDO) is performed to suggest avenues to improve performance over the entire range, as measured by overall fuel consumption.
\end{abstract}

\section{Introduction}

The critical question examined in this paper is that of how to evaluate the performance of highspeed combustors, including isolators and internal expansion components. A complete approach to evaluating highly integrated designs requires an "integrated" method. That is, the evaluation of the design should consider design performance of multiple components over a range of conditions and compute some scalar metric with this information. Previous work, [1], has shown that a vehicle designed to have high pressure recovery factor (PRF) at a single design point will be likely to have poor performance away from that point. Recent work, [1, 2], has attempted to extend this principle into other components with the end goal of using an integrated optimization approach for the entire vehicle, including control and trajectory.

To develop this technique we have concentrated on a few combustor designs available in the literature, which have been examined in experiments, $[3,4,5]$. The performance of these designs as predicted by the MASIV code, [6], has previously been computed in a trade study that considered a range of operating conditions[2]. The present study seeks to expand previous efforts by using mathematical optimization.

It is important to note that the combustors used as a starting point for this optimization are laboratory combustors that were not specifically designed to produce high thrust or efficient operation. They simply provide a reasonable place to start, since they are known to be operable, and they provide data that allow us to validate the model before using it in the optimization study.

We have chosen to examine a space-access trajectory for an airbreathing 2nd stage of a threestage to orbit vehicle. This implies a certain starting point (basically a lower limit for operability of the ram-mode) and stopping point (basically an upper limit for operability of the scram-mode). Ignoring for the moment the various other components required to actually achieve this trajectory (such as lifting the vehicle to the initial Mach number and altitude), we examine how an engine may be optimized for minimal fuel consumption as it flies along a fixed trajectory.

\footnotetext{
${ }^{*}$ Graduate Research Assistant, Department of Aerospace Engineering, AIAA Student Member

$\dagger$ Professor, Department of Aerospace Engineering, AIAA Fellow
} 
Fuel consumption was chosen as the objective function, since this is a key component of vehicle gross weight, hence, cost, of a space access vehicle. A fixed trajectory defined by constant dynamic pressure was chosen for convenience of parametrization. Previous authors, [7], have used similar a trajectory because control surface effectiveness and overall lift are assumed to be well-behaved on a constant dynamic pressure trajectory.

We ignore the possible implications of internal flowpath control systems and other types of variable geometry (such as variable inlets, cowls or nozzles). Recent air and ground tests have concentrated on fixed flowpaths, [8,9]. Of course, the operability range and engine performance can likely be improved by using variable geometry so there is some tradeoff between complexity, weight, and development cost that we neglect for now, [10]. A generic engine flowpath with similar properties to X-43 is shown in Fig. 1.

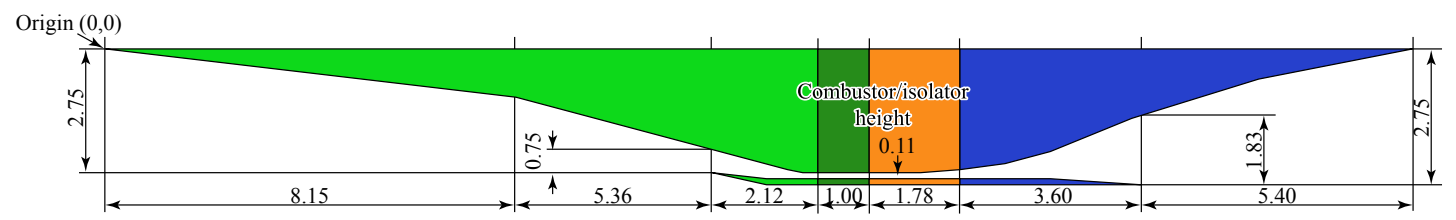

Figure 1. An example vehicle design created using automated design tools.

In this paper, we optimize (i.e.minimize) the fuel consumption of a vehicle having a geometry similar to that of Fig. 1. The axial location of the fuel injector, angle of divergence of the combustor, and axial location of the divergence were the design variables used to optimize the fuel consumption.

\section{Analysis of Available Designs}

In order to obtain a starting point for optimization, let us first consider some designs that are available in the open literature, [3, 11]. These are laboratory scale combustors which are used for tests of combustion processes such as flame holding and combustion efficiency. These are not necessarily designed to generate thrust, but they do provide a reasonable starting point for combustor design because they are known to operate in a stable mode.

Figure 2a shows the geometry of the combustor originally used by Micka et al., [11], which was used to determine some of the constants used in the MASIV model. Another geometry of the same family is shown in Fig. 2b, based on the description given in [12]. Thrust information is not available for either of these geometries.

These combustors were analyzed in a study, [2], that identified a few features of each that contribute to thrust performance. The overall performance of each design was evaluated over a range of Mach numbers $6<M<8$ and altitude from $18 \mathrm{~km}$ to $28 \mathrm{~km}$. Operating maps showing how the differences between the designs contribute to their broad scale performance are shown in Fig.3. That study found that injector location, number of wall divergences, location of divergences, and angle of divergences all contribute to overall thrust of a flowpath over a range of operating points. As can be seen from the operating maps, the flowpath of Fig. 2b produces more thrust than that of Fig. $2 \mathrm{a}$ at every point in the operating map. Using a pointwise average, the thrust in Fig. $3 \mathrm{~b}$ is $7 \%$ greater than the thrust in Fig. 3a over the entire range.

More information about the workings of MASIV, MASTrim and the definitions of flowpaths and design spaces can be found in previous work, $[13,2,6]$ 


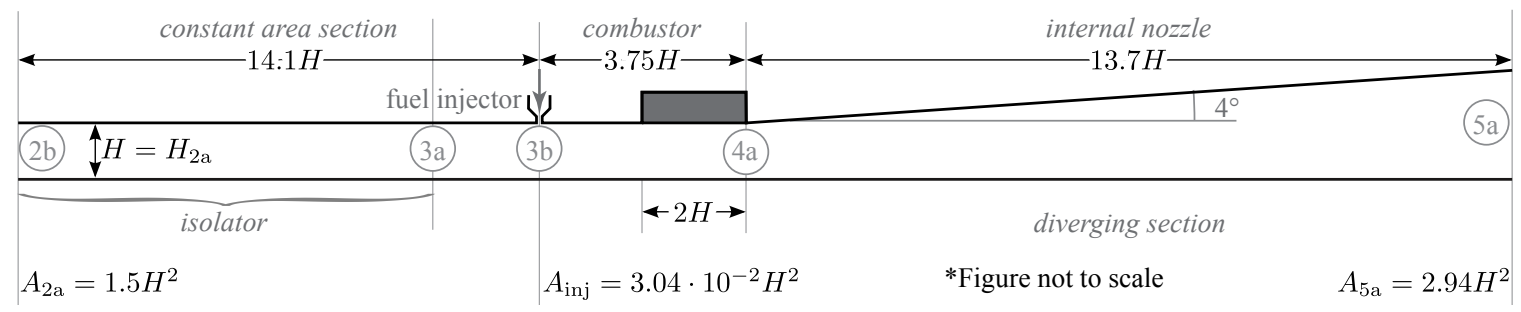

a) The combustor configuration of Micka et al. [11]

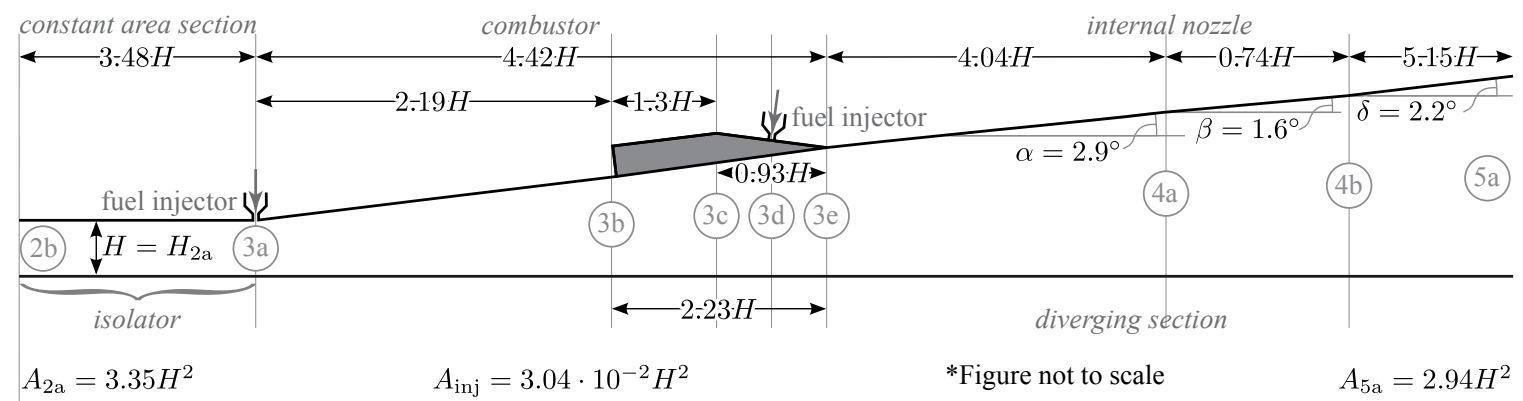

b) The combustor configuration of Donde et al. [12], due originally to Donohue

Figure 2. The two combustors used as design inspiration for the present work.
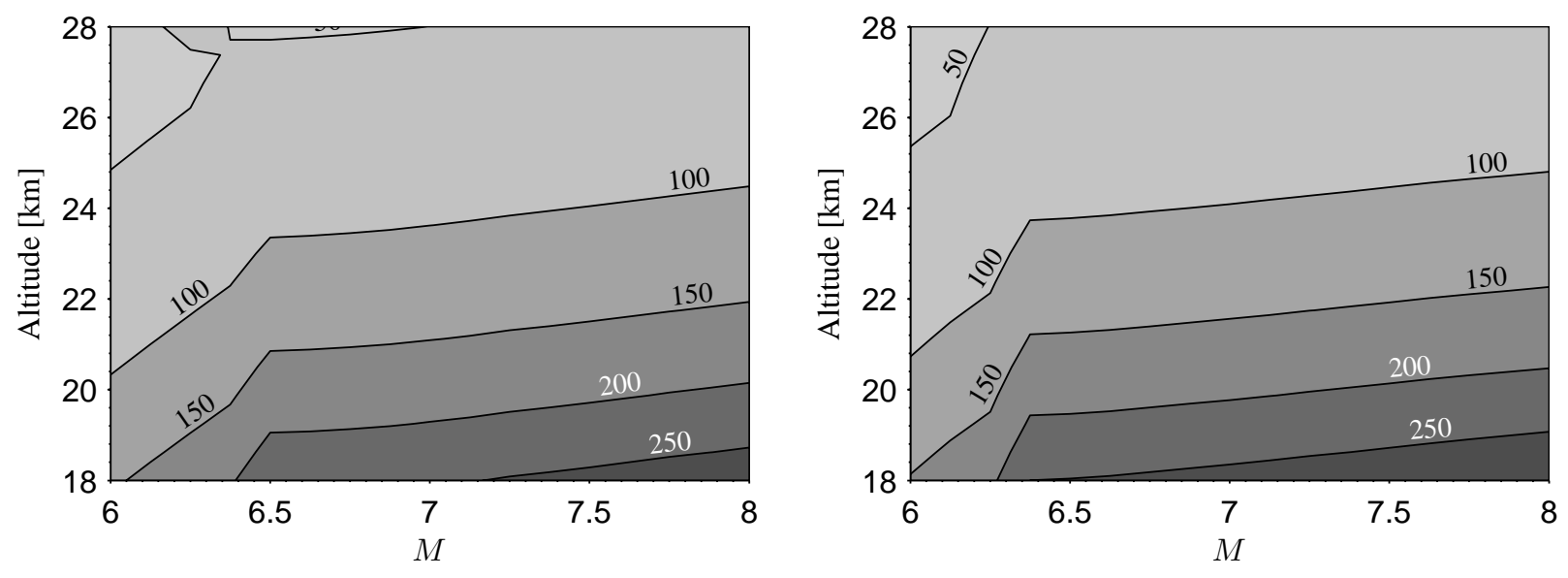

a) Operating map for the geometry shown in Fig. 2a. b) Operating map for the geometry shown in Fig. 2b, but with injectors upstream of the first divergence by $3 H$.

Figure 3. Performance of the baseline combustor and modified combustor of [2] Curves represent different values of thrust in $\mathrm{kN}$. 


\section{Trajectory Definition}

In order to minimize fuel consumption we must define the trajectory we wish the vehicle to follow. Let us define a trajectory as a series of node points at which state variables and control variables are prescribed, $[14,15]$. In general, the vehicle state, $\mathbf{x}$, consists of position and velocity vectors and vehicle orientation:

$$
\mathbf{x}=\left[\begin{array}{llllllllllll}
L & \lambda & h & M & \gamma & \sigma & \alpha & \beta & \phi & P & Q & R
\end{array}\right]^{T}
$$

The position state, $\mathbf{r}$, is defined by longitude, $L$, latitude, $\lambda$, and altitude, $h$ and the velocity state, $\mathbf{v}$, is defined by Mach number, $M$, flight path angle, $\gamma$, and heading angle, $\sigma$. The vehicle orientation is defined by angle of attack, $\alpha$, sideslip angle, $\beta$ and roll angle, $\phi$, and the corresponding angular velocities, $P, Q$, and $R$. The choice of longitude, latitude, and altitude is convenient for very high speed airplanes where Coriolis effects and the curvature of the earth are significant. Also, this selection is consistent with space access trajectory analysis.

For trajectory purposes, we are not particularly interested in the vehicle orientation, only the vehicle's position and velocity. We leave the vehicle orientation states as free parameters, which supplies extra degrees of freedom during computation of the trim state. The "trajectory state" consists of those states of the vehicle which are of interest:

$$
\boldsymbol{\xi}=\left[\begin{array}{llllll}
L & \lambda & h & M & \gamma & \sigma
\end{array}\right]^{T}
$$

Each node of the trajectory also has an associated control state:

$$
\mathbf{u}=\left[\begin{array}{llll}
\delta_{E R} & \delta_{C E} & \delta_{D E} & \delta_{C R}
\end{array}\right]^{T}
$$

The control state variables are fuel equivalence ratio $\delta_{E R}$, common elevator deflection $\delta_{C E}$, defined as the average of the left and right elevator deflection angles, differential elevator deflection $\delta_{D E}$, defined as the deflection angle of the right elevator minus the deflection angle of the left elevator, and common rudder deflection $\delta_{C R}$. We do not allow the rudders to be deflected differentially.

The trajectory and control consist of $n$ arcs, and time $t$ is our independent variable in each leg, $t_{0, i} \leq t \leq t_{f, i}$ for nodes $i=0, \ldots, n$ such that the trajectory state and control state are, [14]

$$
\boldsymbol{\xi}=\boldsymbol{\xi}(t)
$$

Of course, the vehicle parameters $\mathbf{p}$ are also incorporated into the dynamics, so the final equations of motion for the nonlinear vehicle system are [13]

$$
\mathbf{y}=\left[\begin{array}{llllll}
\dot{M} & \dot{\gamma} & \dot{\sigma} & \dot{P} & \dot{Q} & \dot{R}
\end{array}\right]=\mathbf{F}(\mathbf{x}, \mathbf{u}, \mathbf{p})
$$

where the black-box function for the vehicle dynamics, F, is given by the vehicle analysis code (MASTrim, in our case).

It is of interest to note that although most of the vehicle parameters are not functions of time, some of them (e.g. mass or mass distribution, material temperature and properties) may be, and this model allows for such possibilities. Equation (5) is the nonlinear equation of motion for the vehicle. The quantities in Eq. 5 that make up the given state derivative, $\mathbf{y}$, are not the only quantities that could be selected, [13]. However, representing the state in terms of Mach number, flight path angle and heading angle makes sense from a navigation point of view and lends itself to trajectory analysis. Usually we require that $\dot{P}=\dot{Q}=\dot{R}=0$ for stability. 


\section{Trajectory Generation}

Since we are principally concerned with optimizing the combustor design and not the trajectory itself or the control law allowing us to follow that trajectory, we have chosen a single trajectory family for this analysis. In order to fix the mission profile a method is needed to define the trajectory from a given point in the operating space $\left[h_{1}, M_{1}\right]$ to another $\left[h_{2}, M_{2}\right]$. As mentioned earlier, a convenient way to define a curve that connects these two points is to use a constant dynamic pressure curve. This implies that $h_{1}=h\left(M_{1}\right)$ and $h_{2}=h\left(M_{2}\right)$. The 1976 standard atmosphere, [16], was used to perform this lookup.

Using the trajectory nomenclature of Dalle et al., [13], a trajectory was computed which traverses Mach numbers along constant dynamic pressure lines by selecting appropriate altitudes and flight path angles:

$$
\begin{aligned}
W^{n} & =\frac{d h}{d t} \\
\frac{d x^{n}}{d t} & =U^{n} \\
U^{n} & =U \cos \gamma-W \sin \gamma \\
\tan \gamma & =\frac{W^{n}}{U^{n}}
\end{aligned}
$$

Here, $\mathbf{v}^{n}=\left[\begin{array}{lll}U^{n} & V^{n} & W^{n}\end{array}\right]$ are the velocity components in the navigation frame and $\mathbf{v}^{b}=$ $\left[\begin{array}{lll}U & V & W\end{array}\right]$ are the velocity components in the body frame. In order to further constrain the trajectory, we require some reasonable, constant acceleration, $\dot{U}$, of the vehicle.

A simple manipulation yields

$$
\frac{U \cos \gamma \sin \gamma-W \sin ^{2} \gamma}{U \cos \gamma}=\frac{\dot{U}}{a\left\|\mathbf{v}^{b}\right\|} \frac{d h}{d M}
$$

the first approximation of which is

$$
\sin \gamma=\frac{\dot{U}}{a\left\|\mathbf{v}^{b}\right\|} \frac{d h}{d M}
$$

This calculation allows a trajectory to be parametrized by starting and ending Mach numbers, $M_{0}$ and $M_{f}$, a constant dynamic pressure, $q$, and desired forward (body-frame) acceleration, $\dot{U}$.

\section{Performance Estimation}

Our previous study, [2], examined the thrust performance of different designs without regard for their fuel consumption. We now address the question of which combination of properties leads to the lowest overall fuel consumption needed to complete a given mission profile. In order to integrate the performance of the vehicle along each trajectory arc, the Hermite-cubic interpolation with Simpson quadrature method was employed, [15, 17].

In order to exclude controller design and performance from the simulations only quasi-steady solutions to the equations of motion are considered. This means that at each node point the vehicle must be trimmed, that is, $\mathbf{u}$ is chosen such that $\mathbf{y}$ takes on a desired, constant value. This allows us to determine the remaining vehicle and control states that have not been specified at each node. 
Enright and Conway, [15], suggest the (well-known) Hermite-cubic interpolation for the evolution of the vehicle states on each interval $t_{i-1} \leq t \leq t_{i}$ :

$$
\begin{aligned}
\Delta t & =t_{i}-t_{i-1} \\
\boldsymbol{\xi}_{i-\frac{1}{2}} & =\frac{1}{2}\left(\boldsymbol{\xi}_{i-1}+\boldsymbol{\xi}_{i}\right)+\frac{\Delta t}{8}\left[\mathbf{F}\left(\boldsymbol{\xi}_{i-1}, \mathbf{u}_{i-1}\right)-\mathbf{F}\left(\boldsymbol{\xi}_{i}, \mathbf{u}_{i}\right)\right]
\end{aligned}
$$

Equation (13) approximates the trajectory state $\boldsymbol{\xi}_{i-\frac{1}{2}}$ between two known trajectory states $\left(\boldsymbol{\xi}_{i-1}\right.$ and $\boldsymbol{\xi}_{i}$ ), using the known acceleration resultants $\mathbf{y}=\mathbf{F}$ at those states.

Next, we employ Simpson's quadrature to obtain an equation linking states $i-1$ and $i$ through the approximated state $i-\frac{1}{2}$ :

$$
\Delta \boldsymbol{\xi}=\boldsymbol{\xi}_{i-1}-\boldsymbol{\xi}_{i}+\frac{\Delta t_{i}}{6}\left[\mathbf{F}\left(\boldsymbol{\xi}_{i-1}, \mathbf{u}_{i-1}\right)+4 \mathbf{F}\left(\boldsymbol{\xi}_{i-\frac{1}{2}}, \mathbf{u}_{i-\frac{1}{2}}\right)+\mathbf{F}\left(\boldsymbol{\xi}_{i}, \mathbf{u}_{i}\right)\right]
$$

We then impose the constraint that the defect $\Delta \boldsymbol{\xi}$ between states $i-1$ and $i$ be 0 . Thus, (14) can be re-written to give an equation for the desired state derivative at the midpoint of the arc between nodes $i-1$ and $i$ :

$$
\mathbf{y}_{i-\frac{1}{2}}=\frac{3}{2 \Delta t_{i}}\left(\boldsymbol{\xi}_{i}-\boldsymbol{\xi}_{i-1}\right)-\frac{1}{4}\left[\mathbf{F}\left(\boldsymbol{\xi}_{i}, \mathbf{u}_{i}\right)+\mathbf{F}\left(\boldsymbol{\xi}_{i-1}, \mathbf{u}_{i-1}\right)\right]
$$

The computed state derivative $\mathbf{y}_{i-\frac{1}{2}}$ is then used as input to the trim calculation, which in turn solves for the necessary control input $\mathbf{u}_{i-\frac{1}{2}}$ required for the vehicle to achieve the next trajectory point, $\boldsymbol{\xi}_{i}$ :

$$
\mathbf{F}\left(\boldsymbol{\xi}_{i-\frac{1}{2}}, \mathbf{u}_{i-\frac{1}{2}}\right)=\mathbf{y}_{i-\frac{1}{2}}
$$

The quadrature method is used to assure that the control input chosen at the middle of the interval $\left(i-\frac{1}{2}\right)$ is such that it will cause the trajectory state boundary condition at the left- and right-hand conditions to be met, ensuring that the trajectory is met at each given node point in a quasi-steady sense. This frees us from considering the dynamic control problem, but nevertheless allows us to construct and follow trajectories given the assumption that the vehicle performance is relatively well-behaved on each interval and that it would be possible to generate a controller to achieve closed-loop dynamic stability everywhere.

Finally, the fuel consumption over the entire trajectory is the integral of the fuel mass flow rate, $\dot{m}_{\text {fuel }}$, which is known at each node and half-node because it is a function of $\delta_{E R}$ a component of the vehicle control state $\mathbf{u}$. A trapezoidal method is adequate if the values at nodes and half-nodes are used.

This method requires that the trajectory be defined in advance, as described above. In principle the trajectory need not be defined continuously; it could simply be a series of nodes. One reason this is advantageous is that the trajectory itself can be optimized external to the performance calculation routine, with the result being a sequence of discrete nodes.

The general procedure for computing vehicle performance along a given quasi-steady trajectory is the following:

1. Begin with given trajectory $\boldsymbol{\xi} \in\left[\boldsymbol{\xi}_{0}, \ldots \boldsymbol{\xi}_{i}, \ldots, \boldsymbol{\xi}_{n}\right], n=$ number of trajectory arcs.

2. Compute required trim condition at each trajectory node, $\mathbf{y}_{i}=\mathbf{F}\left(\boldsymbol{\xi}_{i}, \mathbf{u}_{i}\right)$, giving $\mathbf{u}_{i}$

3. Interpolate to find midpoint states $\left(\boldsymbol{\xi}_{i-\frac{1}{2}}\right)$ using (13) 
4. Use Simpson quadrature (15) to determine required acceleration at midpoints, $\mathbf{y}_{i-\frac{1}{2}}$

5. Compute trim condition at midpoints to determine required control input $\mathbf{u}_{i-\frac{1}{2}}$ to achieve $\boldsymbol{\xi}_{i}$ beginning from $\boldsymbol{\xi}_{i-1}$ by satisfying $\mathbf{y}_{i-\frac{1}{2}}=\mathbf{F}\left(\boldsymbol{\xi}_{i-\frac{1}{2}}, \mathbf{u}_{i-\frac{1}{2}}\right)$

6. Approximate vehicle performance metric $\Delta m_{\text {fuel }}$ by integrating $\dot{m}_{\text {fuel }}\left(\delta_{E R}\right)$

\section{Parametrization}

In order to optimize the combustor geometry, three parameters were selected based on our earlier examination of existing combustors, [2]. The parameters selected are as follows (Fig. 4):

- Location of fuel injectors with respect to the front of the combustor, $x_{\text {inj }}$

- Location of diverging panel with respect to the front of the combustor, $x_{\text {div }}$

- Angle of diverging panel, $\alpha_{\text {div }}$

Some other important parameters which are not considered in this paper are

- Number of fuel injectors

- Angle of fuel injectors with respect to the wall

- Number of panels in the combustor

- Diameter of fuel injectors

Since some of these parameters take only integer values and cannot vary continuously, we ignore them in order to use a continuous, gradient-based optimization scheme rather than an integer programming environment.

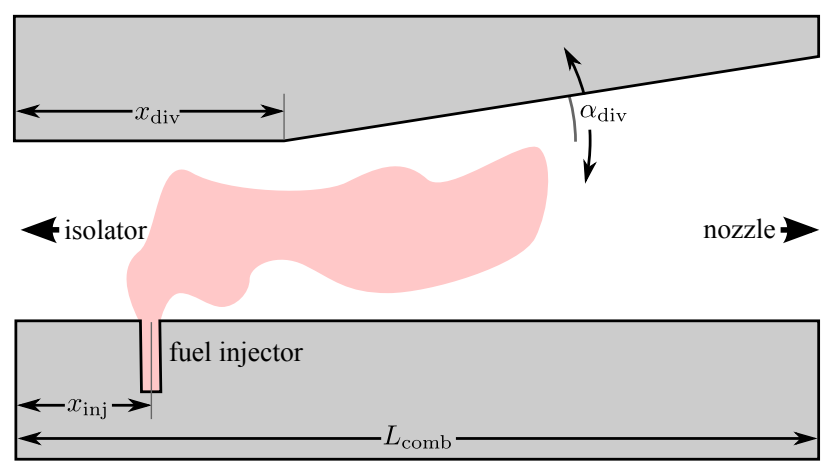

Figure 4. Parameters of interest for a generic 2-D combustor. 


\section{Optimization}

The vehicle is "flown" through a trajectory defined as above, using the 3 vehicle design parameters, $x=\left[\begin{array}{lll}x_{\mathrm{inj}} & \alpha_{\mathrm{div}} & x_{\mathrm{div}}\end{array}\right]$, as the state vector for optimization. The vehicle model is automatically assembled each iteration based on the updated list of design parameters, $x \subset \mathrm{p}$, before the trajectory is flown, as described in [13]. Performance of the design is evaluated based on overall fuel consumption. Thus, the minimization problem can be stated

$$
\begin{aligned}
& \text { minimize } \quad \Delta m_{\text {fuel }}=\int_{t_{0}}^{t_{f}} \dot{m}_{\text {fuel }}(\tau, x) d \tau \\
& x=\left[\begin{array}{lll}
x_{\text {inj }} & \alpha_{\text {div }} & x_{\text {div }}
\end{array}\right]^{T} \\
& \text { subject to } \quad 0<x_{\text {inj }}<L_{\text {comb }} \\
& 0<\alpha_{\text {div }}<\pi / 4 \\
& 0<x_{\text {div }}<L_{\text {comb }}
\end{aligned}
$$

Note that in this optimization, the trajectory defined in (4) is not considered to be a constraint as it would be in an optimal control or trajectory optimization problem. Since the trajectory is satisfied exactly by the solution procedure described above, it can be considered to be part of the evaluation of $\dot{m}_{\text {fuel }, i}$. MATLAB's fmincon routine was employed to find the minimum of Eq.(17), since the actual optimization procedure in this case is relatively simple.

The first trajectory attempted began at $M=5, h=19800 \mathrm{~m}$ (corresponding to the selected dynamic pressure, $q=100 \mathrm{kPa}$ ). We selected an acceleration of $\dot{U}=0.2 \mathrm{~g}$, believing that this was reasonable for this type of vehicle. Interestingly, this simple approach proved to be a failure because the vehicle was unable to trim in ram mode (roughly corresponding to $M<6.5$ for this configuration and trajectory); only the scram cases were able to achieve the required flight path angle and acceleration. Thus, we present scram-only results.

The scram-only section of the trajectory was approximated by $8<M<10,25900 \mathrm{~m}<h<$ $28900 \mathrm{~m}$ (again with $q=100 \mathrm{kPa}$ and $\dot{U}=0.2 \mathrm{~g}$ ). An example of the path of the vehicle on this trajectory is shown in Fig 5. Note that the axes are Mach number and altitude, so angles should not be interpreted as flight path angle or angle of attack.

The scram-mode-only maneuver uses very little fuel compared to the total amount $(\sim 5.7 \%)$, because it is relatively short in duration (just over 5 minutes). Beginning with the vehicle full of fuel is somewhat inconsistent because the mission outlined above is intended to begin at a lower Mach number that might be achieved with a turbojet. However, the baseline vehicle was designed for $M=8,[13,6]$, so this scram-only trajectory corresponds to the baseline configuration.

\section{Results}

Three optimizations were performed with this fixed trajectory. First, only the axial position of the injector, $x_{\text {inj, }}$, was allowed to vary, while $\alpha_{\text {div }}, x_{\text {div }}$, and all other properties of the vehicle and combustor were kept constant. Second, only the axial position of the injector, $x_{\text {inj }}$, and the divergence angle of the panel $\alpha_{\text {div }}$, were allowed to vary, while $x_{\text {div }}$ and all other properties of the vehicle and combustor were kept constant. Third, all three of the optimization state variables (the axial position of the injector, $x_{\text {inj }}$, the divergence angle of the panel $\alpha_{\text {div }}$, and the axial position of the front of the panel, $x_{\text {div }}$ ) were allowed to vary, while all other properties of the vehicle and combustor were kept constant. 


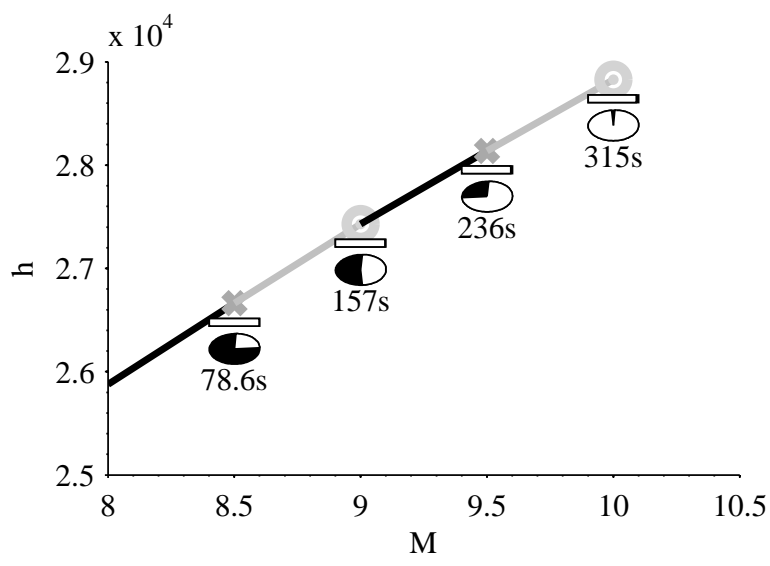

Figure 5. Scram-mode-only trajectory path through $M-h$ space, with $h$ in $\mathrm{m}$. The rectangular bars show fuel consumed compared to overall fuel ( $22700 \mathrm{~kg}$ in this case) and the circles show time elapsed compared to total maneuver time, with elapsed time printed below. Points marked with circles are trajectory nodes, $(i)$; points marked with $x$ 's are trajectory arc midpoints, $\left(i-\frac{1}{2}\right)$.

Table 1. Scram-mode-only optimization: fine tolerances

\begin{tabular}{cccc}
\hline \hline Variable & baseline value & 1 var. optimized & 2 var. optimized \\
\hline$x_{\text {inj }}$ & $0.166 \mathrm{~m}$ & $0.036 \mathrm{~m}$ & $0.058 \mathrm{~m}$ \\
$\alpha_{\text {div }}$ & $3.99^{\circ}$ & $3.99^{\circ}$ & $3.04^{\circ}$ \\
$x_{\text {div }}$ & $0.831 \mathrm{~m}$ & $0.831 \mathrm{~m}$ & $0.831 \mathrm{~m}$ \\
$\Delta m_{\text {fuel }}$ & $1299 \mathrm{~kg}$ & $1252 \mathrm{~kg}$ & $1242 \mathrm{~kg}$ \\
$\%$ improvement & 0 & 3.5 & 4.4 \\
\hline \hline
\end{tabular}


None of the constraints was active in any of the optimizations. However, the upstream limit of fuel injector location at 0 was invoked because placing the injector farther upstream would cause some of the fuel to diffuse upstream out of the combustor domain, where it is not accounted for in the simulation. In effect, this means that the preferred injector position for scram operation was as far upstream as allowed by the vehicle design, presumably because this gives greater compression due to confinement in the constant-area section of the combustor.

Using $42.4 \mathrm{GHz}$ processors (a quad-core Intel Xeon processor with MATLAB parallel toolbox), these simulations ran in 5.4 hours and 16 hours for the one- and two-variable cases, respectively. The optimization design vector $(x)$ tolerance was $10^{-6}$ for each case, and the tolerance on the search direction was $2 \cdot 10^{-6}$. The three-variable case was not run at tight tolerances because the run time was expected to be excessive. Results for these cases are summarized in Table 1.

Table 2. Scram-mode-only optimization: coarse tolerances

\begin{tabular}{ccccc}
\hline \hline Variable & baseline value & 1 var. optimized & 2 var. optimized & 3 var. optimized \\
\hline$x_{\text {inj }}$ & $0.166 \mathrm{~m}$ & $0.042 \mathrm{~m}$ & $0.038 \mathrm{~m}$ & $0.042 \mathrm{~m}$ \\
$\alpha_{\text {div }}$ & $3.99^{\circ}$ & $3.99^{\circ}$ & $2.84^{\circ}$ & $3.56^{\circ}$ \\
$x_{\text {div }}$ & $0.831 \mathrm{~m}$ & $0.831 \mathrm{~m}$ & $0.831 \mathrm{~m}$ & $0.865 \mathrm{~m}$ \\
$\Delta m_{\text {fuel }}$ & $1299 \mathrm{~kg}$ & $1254 \mathrm{~kg}$ & $1233 \mathrm{~kg}$ & $1239 \mathrm{~kg}$ \\
$\%$ improvement & 0 & 3.5 & 5.1 & 4.6 \\
\hline \hline
\end{tabular}

We also ran simulations with coarser tolerances on each of the variables. The coarse tolerance simulations ran in 1.7 hours, 4.4 hours, and 12.5 hours for the one-, two-, and three-variable cases, respectively. The function $\left(\Delta m_{\text {fuel }}\right)$ tolerance was $10^{-3}$, the optimization design vector $(x)$ tolerance was $10^{-4}$, and the tolerance on the search direction was $2 \cdot 10^{-4}$ for each coarse case. Note that results for the coarse tolerance cases were equally acceptable as fine tolerance results for judging the merits of this approach, and much less expensive. The reason for the apparent abnormality for the two-variable, coarse tolerances case having lower fuel consumption than the three-variable, coarse tolerances case is unknown.

Finally, the operating map of the optimized combustor is shown alongside the previous two examples in Fig. 6. Note that the performance of the combustor optimized using a single panel is better than the baseline (Fig. 2a) by $3 \%$, while the performance of the combustor shown in Fig. $2 b$ is $7 \%$ better than the baseline (by pointwise average). This indicates that combustor shape accounts for much of the thrust performance, which is to be expected. It is not clear how combustor shape affects fuel consumption over the entire trajectory, but shape should certainly be included in future investigations.

\section{Conclusions}

In this paper, we integrated the MASIV and MASTrim low-order models were integrated into a design optimization analysis for a dual mode ramjet-scramjet flowpath. We found that a combustor flowpath that was designed to properly stabilize flames in ram mode in laboratory experiments was able to generate sufficient thrust to trim a vehicle along a scram-mode-only trajectory from $M=8$ to $M=10$. Using conventional trajectory analysis methods and readily-available optimization routines, we were able to determine values of $x=\left[\begin{array}{lll}x_{\mathrm{inj}} & \alpha_{\mathrm{div}} & x_{\mathrm{div}}\end{array}\right]$ that minimize fuel consumption. It is interesting to note that combustor designs with divergence angles of $2^{\circ}$ to $4^{\circ}$ are common in practice. 

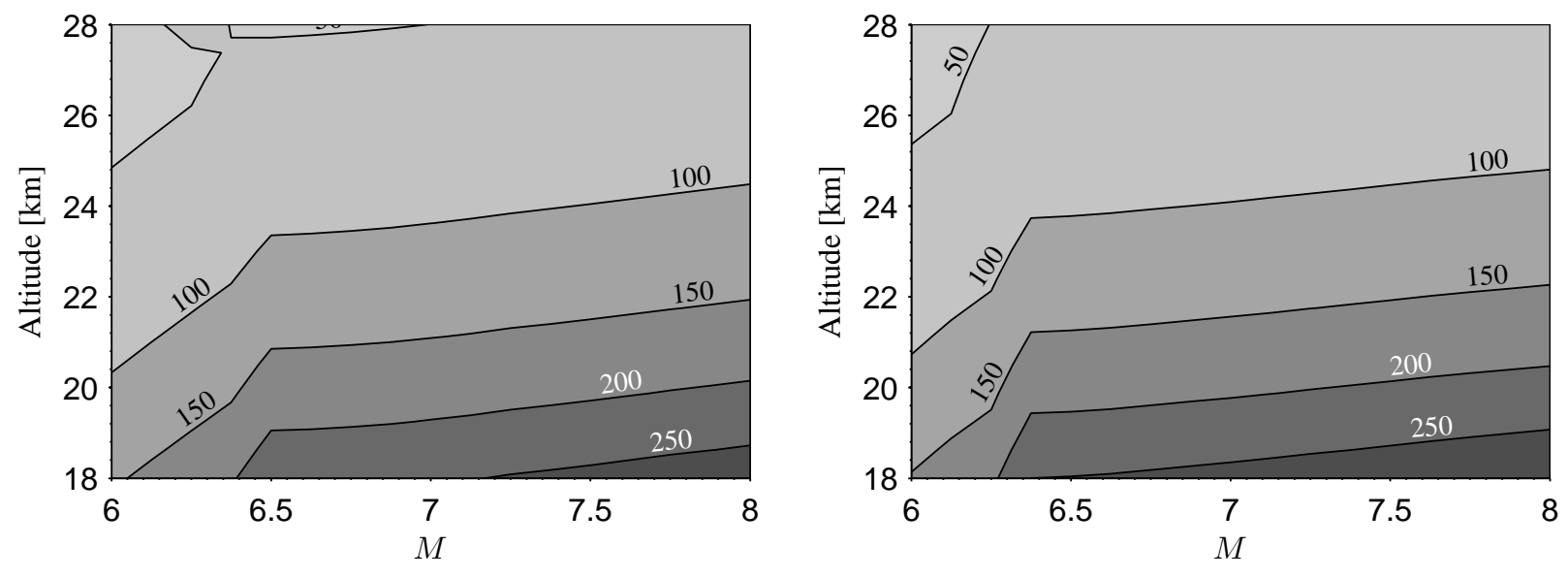

a) Operating map for the geometry shown in Fig. 2a. b) Operating map for the geometry shown in Fig. 2b, but with injectors upstream of the first divergence by $3 H$.

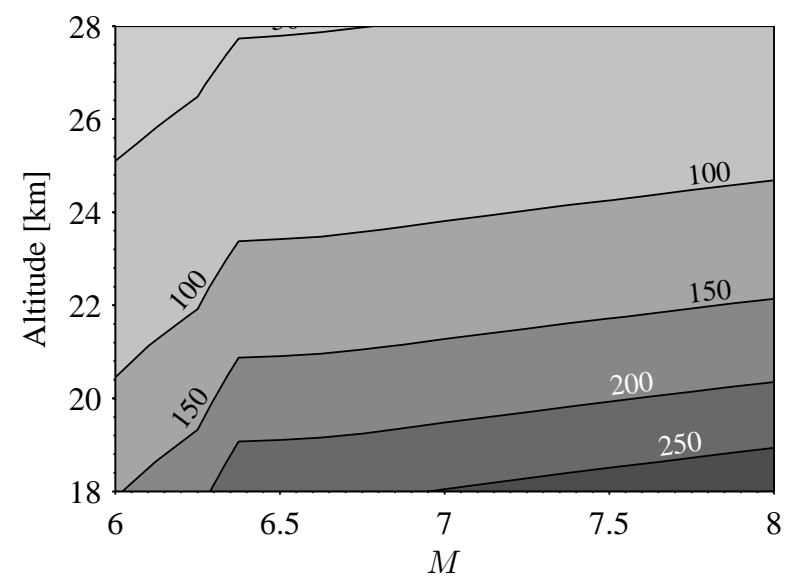

c) Operating map for the geometry of the three-variable case, coarse tolerances.

Figure 6. Performance of the baseline combustor and modified combustor of [2], along with performance of the optimized geometry. Curves represent different values of thrust in $\mathrm{kN}$. 
We also found that a dual-mode combustor that produces adequate thrust for a vehicle when operating in scram mode may not always produce adequate thrust when operating in ram mode. This difficulty is exhibited by the fact that our baseline vehicle is incapable of trimming in the ram mode, so it cannot achieve the scram section of the trajectory when beginning at $M=5$.

This investigation supports the conclusion that scram-mode operation of combustors is enhanced when fuel injection occurs as far forward in the flowpath as is practical. Since this paper only considers scram-mode operation due to the difficulties discussed above, this result necessarily ignores isolator length requirements due to pre-combustion shock train length.

Since this optimization found that the divergence angle of the single panel in the combustor did not trend to either of the bounds $\left(0^{\circ}\right.$ and $\left.45^{\circ}\right)$, and since a three-panel combustor was previously found to have better thrust than a single panel combustor (including the optimized geometry used here), we also conclude that the detailed shape of the combustor is important. It seems insufficient only to consider overall area expansion ratio, even when operating exclusively in scram mode.

\section{References}

[1] Torrez, S. M., Driscoll, J. F., Dalle, D. J., and Fotia, M. L., "Preliminary Design Methodology for Hypersonic Engine Flowpaths," 16th AIAA/DLR/DGLR International Space Planes and Hypersonic Systems and Technologies Conference, 2009, AIAA Paper 2009-7289.

[2] Torrez, S. M., Dalle, D. J., and Driscoll, J. F., "Design of Dual-Mode Hypersonic Vehicle Flow Paths Using a Reduced-Order Model," 17th AIAA International Space Planes and Hypersonic Systems and Technologies Conference, April 2011.

[3] Mathur, T., Streby, G., Gruber, M., Jackson, K., Donbar, J., Donaldson, W., and Jackson, T., "Supersonic Combustion Experiments with a Cavity Based Fuel Injector," 1999, AIAA 99-2102.

[4] Micka, D. J., Torrez, S. M., and Driscoll, J. F., "Measurements and Modeling of the Heat Release Distribution in a Dual-mode Scramjet Combustor with Wall Fuel Injection," Proceedings of the 6th U.S. National Combustion Meeting, 2009.

[5] Lin, K.-C., Ryan, M., Carter, C., Gruber, M., and Raffoul, C., "Raman Scattering Measurements of Gaseous Ethylene Jets in Mach 2 Supersonic Crossflow," Journal of Propulsion and Power, Vol. 26, No. 3, 2010, pp. 503-513.

[6] Torrez, S. M., Driscoll, J. F., Ihme, M., and Fotia, M. L., "Reduced Order Modeling of Turbulent Reacting Flows With Application to Scramjets," Journal of Propulsion and Power, Vol. 27, No. 2, March-April 2011, pp. 371-382.

[7] Bolender, M. A., Oppenheimer, M. W., and Doman, D. B., "Effects of Unsteady and Viscous Aerodynamics on the Dynamics of a Flexible Air-breathing Hypersonic Vehicle," AIAA Atmospheric Flight Mechanics Conference and Exhibit, 2007, AIAA Paper 2007-6397.

[8] Jackson, K. R., Gruber, M. R., and Barhorst, T. F., "The HIFiRE Flight 2 Experiment: An Overview and Status Update," 45th AIAA/ASME/SAE/ASEE Joint Propulsion Conference \& Exhibit, August 2009, AIAA 2009-5029.

[9] Hank, J. M., Murphy, J. S., and Mutzman, R. C., "The X-51A Scramjet Engine Flight Demonstration Program," 15th AIAA International Space Planes and Hypersonic Systems and Technologies Conference, 2008, AIAA Paper 2008-2540. 
[10] Bouchez, M., Perillat, V., Avrashkov, V., and Kopchenov, V., "Numerical and experimental scientific investigation of combustion in a translating cowl dual-mode ramjet," 2011, AIAA Paper 2011-2313.

[11] Micka, D. J. and Driscoll, J. F., "Combustion characteristics of a dual-mode scramjet combustor with cavity flameholder," Proceedings of the Combustion Institute, Vol. 32, 2009, pp. 2397-2404.

[12] Donde, P., Koo, H., and Raman, V., "A Multivariate Quadrature Based Moment Method for Supersonic Combustion Modeling," 49th AIAA Aerospace Sciences Meeting including the New Horizons Forum and Aerospace Exposition, January 2011, AIAA 2011-322.

[13] Dalle, D. J., Torrez, S. M., Driscoll, J. F., and Bolender, M. A., "Flight Envelope Calculation of a Hypersonic Vehicle Usinga First Principles-Derived Model," 17th AIAA International Space Planes and Hypersonic Systems and Technologies Conference, 2011.

[14] Betts, J. T., "Survey of Numerical Methods for Trajectory Optimization,” Journal of Guidance, Control, and Dynamics, Vol. 21, 1998, pp. 193-207.

[15] Enright, P. J. and Conway, B. A., "Discrete Approximations to Optimal Trajectories Using Direct Transcription and Nonlinear Programming," Journal of Guidance, Control, and Dynamics, Vol. 15, No. 4, July-August 1992, pp. 994-1002.

[16] U. S. Standard Atmosphere, 1976, U. S. Government Printing Office, October 1976, NOAA-S/T 761562.

[17] Kunz, K. S., Numerical Analysis, McGraw-Hill, 1957. 